\title{
¿CÓMO TRABAJAR LAS COLOCACIONES LATINAS EN EL AULA? UNA PROPUESTA A PARTIR DE SUS CARACTERÍSTICAS COMBINATORIAS, SEMÁNTICAS Y SINTÁCTICAS
}

\author{
María Isabel Jiménez Martínez - Eveling Garzón Fontalvo \\ Universidad Nacional Autónoma de México - Universidad Autónoma de Madrid \\ eirenez@hotmail.com - eveling.garzon@uam.es
}

\begin{abstract}
RESUMEN
El objetivo de este artículo es doble: por una parte, presentar las principales características combinatorias, semánticas y sintácticas de las colocaciones en latín y, por otra, aportar una serie de explicaciones y ejercicios que permitan a los estudiantes entender su funcionamiento. Para ello, partiremos de la naturaleza restringida de este tipo de combinaciones de palabras. A continuación, profundizaremos en la idea de que son los nombres, y no los verbos, su núcleo semántico. Y, por último, analizaremos la particular contribución sintáctica de cada uno de estos elementos al conjunto. Toda la explicación teórica de nuestra propuesta se acompañará de una serie de actividades prácticas cuyo fin es aprender a identificar, entender y traducir algunas colocaciones con los verbos facere, habere y dare.
\end{abstract}

PALABRAS ClAVE: colocaciones, latín, semántica, sintaxis, didáctica.

HOW TO APPROACH LATIN COLLOCATIONS IN THE CLASSROOM?

A PROPOSAL BASED ON THEIR COMBINATORY, SEMANTIC AND SYNTACTIC FEATURES

\section{ABSTRACT}

The aim of this article is twofold: on the one hand, to highlight the main combinatory, semantic and syntactic features of the collocations in Latin and, on the other hand, to provide a series of explanations and exercises that allow students to understand how they work. To do so, first we will focus on the restricted nature of this type of word combinations. Next, we will go deeper into the idea that the nouns, and not the verbs, are the semantic core of these constructions. And finally, we will analyze the syntactic contribution of each of these elements to the whole. All the theoretical explanation of our proposal will be accompanied by a series of practical activities focused on learning to identify, understand, and translate some collocations with the verbs facere, habere and dare.

KeYWORDS: collocations, Latin, Semantics, Syntax, Didactics.

\section{INTRODUCCIÓN}

Una de las principales dificultades a las que se enfrenta cualquier estudiante de una segunda lengua consiste en aprender no solo sus normas gramaticales 
y los significados de sus palabras, sino también el manejo correcto de su léxico, en especial de las locuciones y las colocaciones, es decir, las combinaciones que, de forma frecuente, preferente e idiosincrásica, emplea una comunidad lingüística para referirse a ciertas realidades ${ }^{1}$. Estas combinaciones preferentes de palabras pueden adoptar distintos esquemas sintácticos (Koike, 2001; Mel'cuk, 1995): sustantivo + adjetivo (por ejemplo, dinero negro o dulcis amor 'amor dulce'), verbo + adverbio (enamorarse perdidamente o dulce ridere 'reír dulcemente'), verbo + nombre (tener celos o inuidiam habere 'tener envidia'), entre otros. En este trabajo nos interesan las colocaciones formadas por un sustantivo y un verbo, del tipo de dar las gracias, tener sed o tomar precauciones en español y, más concretamente, aquellas del latín como impetum facere 'hacer un ataque', curam habere 'tener cuidado' o gratias agere 'dar las gracias'.

Ya en la antigüedad un escritor como Varrón (s. II-I a.C.) explicó ciertas combinaciones preferentes de palabras con verbos de significado próximo (facere, agere y gerere) y consideró que el hecho de conocerlas y emplearlas de forma adecuada era señal de un buen conocimiento del latín, como se muestra en el pasaje (1):

[...] propter similitudinem agendi et faciendi et gerendi quidam error his qui putant esse unum. Potest enim aliquid facere et non agere, ut poeta facit fabulam et non agit, contra actor agit et non facit, et sic a poeta fabula fit, non agitur, ab actore agitur, non fit (Varro ling. 6.77-78).

«[...] por la similitud existente entre agere, facere y gerere, algunos han incurrido en el error de considerar que son una misma cosa. En efecto, se puede facere algo y no agere, por ejemplo, un autor compone (facit) una obra dramática, pero no la representa él (agit); y a la inversa, un actor la representa (agit), pero no la compone (facit); del mismo modo, una obra teatral es realizada ( $f i t)$ por un autor, pero no es representada (agitur) por él: es representada (agitur) por el actor, pero no realizada (fit) por él» (Varrón, trad. 1980).

Sin embargo, quizás porque en las lenguas de corpus no disponemos de hablantes nativos que nos ayuden a determinar lo que es idiomático, este tipo de construcciones no han sido consideradas un objeto de estudio específico en latín hasta hace poco menos de una década ( $c f$. Baños, 2012, 2016; Garzón y Jiménez, 2018; Jiménez, 2016; Mendózar, 2015; Pompei, 2016; Tur, 2019, entre otros) ${ }^{3}$. Desde entonces se han analizado aspectos relacionados con ellas tanto en el interior de la lengua latina como en comparación con otras (especialmente el griego antiguo y las lenguas romances) $)^{4}$.

${ }^{1}$ Sobre la didáctica de las colocaciones, $c f$. Lewis (2000).

${ }^{2}$ Las colocaciones han sido objeto de un enorme interés en la lingüística moderna desde mediados del siglo pasado. Algunos estudios relevantes en lenguas modernas son Alonso (2004), Gross (1981), Gross (2004), entre otros.

${ }^{3}$ Para un exhaustivo estado de la cuestión acerca de las colocaciones en latín, $c f$. Mendózar (2020).

${ }^{4}$ Prueba de ello son, por una parte, la Journée d'étude Les Constructions à verbe support en latin, llevada a cabo en Toulose (23-24 de septiembre de 2016), seguida de la publicación de las actas ( $c f$. Bodelot y Spevak [dirs.], 2018); y, por otra, el proyecto de investigación DiCoLat, que busca la creación 
Esto se observa con claridad si echamos un vistazo a algunos manuales de sintaxis latina publicados en español. En ellos, tal como vamos a ilustrar a continuación, estas combinaciones aparecen solo de forma anecdóticas.

Para comenzar, la Introducción a la sintaxis estructural del latín de Rubio (1982) hace referencia indirecta a este tipo de construcciones en el apartado dedicado al caso acusativo, ya que las considera un ejemplo no prototípico de "acusativo interno»: «Aunque no se dé la figura etimológica, no es menos «interno» el acusativo cuando nombre y verbo -sin coincidencia etimológica- cubren más o menos exactamente la misma zona semántica: proelium pugnare 'luchar una batalla', aetatem uiuere 'vivir una etapa', longam ire niam 'hacer un largo camino', etcétera» (Rubio, 1982: 122).

Del mismo modo, en la Sintaxis Latina de Bassols ([1956] 1992) no se dedica un apartado específico a las colocaciones que nos ocupan, aunque se utilizan abundantes ejemplos en los que aparecen y se comentan parcialmente -también a propósito del acusativo interno- como sigue: «El acusativo no deriva de la misma raíz que el verbo pero tiene un significado afín; por ej.: pugnam proeliare 'luchar una batalla', aevum vivere 'vivir una etapa', errorem insanire 'enloquecer', etcétera» (Bassols, [1956] 1992: 31). Además, en este manual se consideran estas construcciones muestras de un lenguaje popular, poético o de preceptos y leyes.

Encontramos la misma situación en la Sintaxis del latín clásico coordinada por Baños (2009), donde se mencionan solo de forma tangencial en el apartado dedicado al acusativo interno (Baños, 2009: 140-141). Sin embargo, en la reedición de esta sintaxis - prevista para este año-la referencia a las colocaciones es ya frecuente, tal como lo es en la monumental The Oxford Latin Syntax de Pinkster (2015).

En la misma línea de los manuales de sintaxis, cabe notar que las colocaciones apenas si son abordadas en las clases de lengua latina de Bachillerato, a juzgar por el tratamiento que reciben en los libros de texto. Así pues, tras analizar tres libros de editoriales diferentes para primero de Bachillerato -García Gual et al. (2010), Edebé (2011) y Sanchis (2012) - encontramos que estas construcciones no se explican. En concreto, por lo que respecta al primer libro, podemos señalar que, aunque existen ejercicios de traducción en los que aparecen colocaciones -ya en las primeras lecciones donde se explican los casos (cf. Gn. Pompeius bellum gerebat 'Cneo Pompeyo hacía la guerra')-, no se da ninguna pista a los estudiantes de cómo debería traducirse este tipo de estructuras. De forma similar, en Edebé (2011) también se documentan colocaciones en los textos que se deben traducir al español ( $c f$. sed in itinere hostes in nostros impetum fecerunt 'pero en el camino los enemigos nos atacaron') sin que

de un diccionario en línea de colocaciones latinas. Respecto al estudio comparativo de las colocaciones latinas con otras lenguas, pueden consultarse, por ejemplo, Baños y Jiménez (2017), Jiménez y Melis (2018) y López (2019).

${ }^{5}$ Quizá este hecho se deba a que este fenómeno era considerado hasta bien entrado el s. XX parte de una lengua descuidada y vulgar y, por tanto, era abordado más desde la estilística que desde la sintaxis propiamente ( $c f$. Hofmann, 1958). 
se dé una explicación sobre estas. Por último, en Sanchis (2012) el tratamiento que recibe una colocación del tipo bellum gerere se limita a su aparición en el vocabulario de la unidad, dentro de la entrada de gero, de la siguiente manera: gero, -is, -ere. llevar, llevar a cabo (bellum gerere: hacer la guerra).

Con todo, el hecho de que estas construcciones aparezcan en los libros de texto -aunque de manera puntual- guarda una relación directa con su documentación en algunas de las pruebas de Evaluación para el Acceso a la Universidad (EvAU). A manera de ejemplo, podemos observar los siguientes textos correspondientes a las pruebas de 2020 de las comunidades autónomas de Extremadura y Asturias (las colocaciones se señalan en negrita):

(2) Dum bellum in Numidia contra Iugurtham geritur, consules M. Manlius et Q. Caepio a Cimbris et Teutonis et Tugurinis et Ambronibus, quae erant Germanorum et Gallorum gentes, victi sunt iuxta flumen Rhodanum et ingenti internecione; etiam castra sua perdiderunt (Prueba de Evaluación del Bachillerato para el Acceso a la Universidad, 2020, Extremadura).

(3) Erant hae difficultates belli gerendi quas supra ostendimus, sed tamen multa Caesarem ad id bellum incitabant: iniuria retentorum equitum Romanorum, rebellio facta post deditionem, defectio obsidibus, tot civitatum coniuratio... (Prueba de Evaluación del Bachillerato para el Acceso a la Universidad, 2020, Asturias).

Hasta aquí, hemos reflejado, por una parte, la presencia relativamente frecuente de las colocaciones en latín y, por otra, la ausencia de explicaciones específicas sobre ellas en las gramáticas y en los libros de texto analizados. Una vez identificada esta carencia, el objetivo concreto de este artículo es ofrecer una serie de actividades que permitan acercar a los estudiantes de niveles iniciales de latín a este tipo de construcciones ${ }^{6}$; en este sentido, consideramos que el estudio de este fenómeno de la lengua puede contribuir a una mejor comprensión y traducción de los textos clásicos.

Así las cosas, la estructura propuesta para este trabajo es la siguiente: primero, presentamos una caracterización de las colocaciones $(\$ 2)$; a continuación, describimos nuestra propuesta teórica y práctica para abordar estas combinaciones en el aula (\$3); y, por último, recapitulamos los principales aportes de este artículo (\$4).

\section{CARACTERIZACIÓN DE LAS COLOCACIONES EN LATÍN}

Una de las características más importantes de las colocaciones es que presentan un cierto grado de fijación e idiomaticidad en la lengua. Para explicar mejor

${ }^{6}$ Es importante mencionar en este punto el reciente trabajo de Tur (2020), en el que se ofrece una aproximación a la enseñanza de las colocaciones latinas desde un enfoque cognitivo. 
esto, representamos linealmente diferentes tipos de combinaciones de palabras a través de la Figura 1:
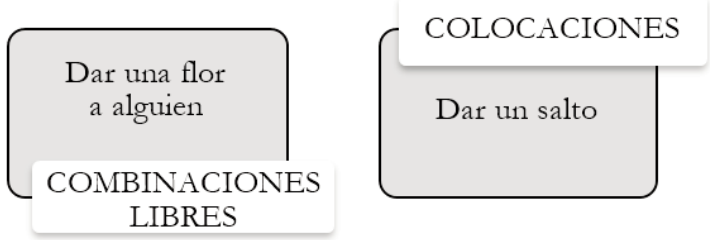

Fijación

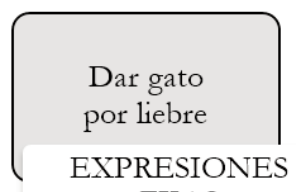

FIJAS

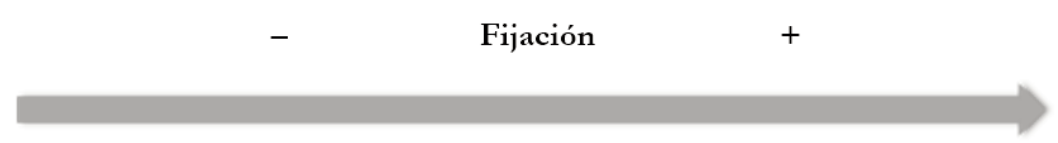

Figura 1. Esquema del grado de fijación presentado por las colocaciones ${ }^{7}$.

En un extremo de esta línea hemos colocado las combinaciones libres de palabras - dar una flor a alguien-, en las que la aparición de los términos es fruto de la relación puntual de sus significados y no se entienden como un todo unitario; y, en el otro extremo, las expresiones fijas - dar gato por liebre-, que están prácticamente fosilizadas. Las colocaciones, en este caso dar un salto, se sitúan entre ambos polos, puesto que no están tan fijadas como las unidades fraseológicas, pero tampoco son fortuitas, sino que son combinaciones preestablecidas en la conciencia de los hablantes. De este modo, los hablantes de una determinada comunidad lingüística han aprendido que, dada una palabra -salto-, hay una o un número reducido de ellas con las que se puede combinar-dar-. Esto quiere decir, grosso modo, que las colocaciones son producto de ciertas restricciones combinatorias.

Como ya hemos indicado, las combinaciones que ocupan nuestra atención en este trabajo son las colocaciones verbo-nominales con el nombre en función de Objeto (dar un salto o rebellionem facere 'hacer una rebelión') por ser las más productivas y mejor estudiadas en la bibliografía de las lenguas modernas. principales:

A continuación, presentaremos muy brevemente algunos de sus rasgos

a. Significado (semi)idiomático: las colocaciones son propias de una determinada comunidad lingüística y, en muchas ocasiones, cuando las traducimos, no lo podemos hacer literalmente, puesto que la lengua de origen y la de destino no comparten siempre los mismos referentes: por poner solo un ejemplo sencillo, los paseos, que en español se dan (dar un paseo), en italiano, francés y catalán se hacen (fare una

\footnotetext{
${ }^{7}$ Esta figura y todas las tablas que presentamos son de elaboración propia.
} 
passeggiata, faire une promenade y fer un passeig) y en inglés se toman (take a walk). Para expresar esta misma idea, el latín clásico utiliza el verbo simple ambulare.

b. Verbo simple equivalente: estas construcciones constituyen perífrasis con las que se hace referencia a una unidad de sentido, por lo que, a menudo, cuentan con un verbo simple equivalente semántica y sintácticamente, como hacer una promesa/prometer o, en latín, pugnam facere/pugnare 'luchar' y uulnus dare/uulnerare 'herir'.

c. Nombre como núcleo de la construcción: dentro de este tipo de combinaciones es el nombre, y no el verbo, el que aporta la mayor carga semántica y selecciona los complementos. De esta forma, en una colocación como impetum facere 'hacer un ataque', que se construye generalmente con un sintagma preposicional in + Acusativo ('hacer un ataque contra X'), es impetum el elemento predicativo preponderante del que depende el complemento preposicional (in + Acusativo).

Es precisamente este último criterio el que sirve de base para nuestra propuesta didáctica $(\$ 3)$. Así, para abordar en el aula el concepto de colocación nos centraremos en la idea de que en este tipo de construcciones "la tarea de predicar se invierte» (De Miguel, 2006). ¿Qué quiere decir esto? Cuando los estudiantes empiezan a estudiar una segunda lengua suelen hacerlo a partir de gramáticas tradicionales que, por lo general, se basan en la descripción de las categorías sintácticas definidas por filólogos de la antigüedad grecolatina. Según estas gramáticas, el elemento predicativo por excelencia de la oración es el verbo, puesto que selecciona los diferentes constituyentes que funcionan como sus complementos; por ejemplo, en una oración como Juan llevó a su hija al jardin, los complementos a su hija y al jardín son regidos, esto es, seleccionados, por el verbo llevar.

Sin embargo, las colocaciones no responden a esta dinámica: en ellas el núcleo es el nombre predicativo. Esta situación se ilustra bien en las colocaciones latinas recordationem habere 'recordar' y conspirationem facere 'hacer una conspiración', donde los nombres recordationem 'recuerdo' y conspirationem 'conspiración' tienen una carga semántica muy alta: en este caso concreto, los dos son nombres deverbales que han heredado la estructura semántica de sus verbos base recordor 'recordar' y conspiro 'conspirar' y, por tanto, resulta explicable que sean el núcleo de la construcción. Por su parte, los verbos habere 'tener' y facere 'hacer' presentan una carga semántica mucho más baja: ambos son muy polisémicos y generales, como lo demuestra el hecho de que sus respectivas entradas en el Oxford Latin Dictionary presenten un número altísimo de acepciones (27 para habeo y 30 para facio). Estos verbos muestran, por así decirlo, un debilitamiento de su significado prototípico y de sus funciones como verbos plenos. La función que desempeñan en la colocación es, en esta medida, la de ser «auxiliares» del nombre, de ahí su denominación usual como verbos de apoyo o soporte . $^{8}$.

${ }^{8}$ Empleamos el término verbo soporte por ser la denominación más extendida actualmente, aunque se usan otras como verbos de apoyo, light verbs, operator verbs o Funktionsverben, etiquetas todas ellas que inciden en la idea de que son un tipo de verbos auxiliares que pierden parte de su significado originario y «convierten en predicados a los sustantivos con los que se construyen» (Baños, 2012: 38). 
Con esta descripción teórica como telón de fondo, es el momento ahora de pasar al desarrollo de nuestra propuesta didáctica.

\section{PROPUESTA DIDÁCTICA: ACTIVIDADES PARA APROXIMARSE A LAS COLOCACIONES}

La propuesta que presentamos está destinada a estudiantes de Bachillerato y latín elemental de nivel universitario. Para comenzar, describiremos una serie de actividades que se centran en el concepto de restricción combinatoria y que nos permitirán familiarizar a los estudiantes con la identificación de las colocaciones $(\$ 3.1)$. A continuación, presentaremos dos posibles vías para estudiar estas construcciones en el aula teniendo en cuenta la relación inversa que manifiestan nombres predicativos y verbos de apoyo: una vía, la más importante, basada en la distinta aportación semántica de nombres y verbos $(\$ 3.2)$ y la otra enfocada en la particular contribución sintáctica de cada uno de estos elementos (\$3.3). En este punto, es importante señalar que esta propuesta no deja de ser una primera toma de contacto de los estudiantes con este tipo de unidades léxicas, de ahí que partamos de colocaciones sencillas con tres de los verbos más frecuentes en latín, facere, habere y dare. El objetivo a largo plazo es, como afirma Higueras (1996), ir ampliando poco a poco la red de construcciones para que resulte más fácil almacenarlas y recuperarlas.

\subsection{PRIMEROS PASOS: LAS COLOCACIONES A PARTIR DE LA NOCIÓN DE RESTRICCIÓN COMBINATORIA}

En la fase inicial de nuestra propuesta didáctica nos apoyaremos en uno de los aspectos sobre el que hay mayor consenso en la bibliografía especializada: el hecho de que las colocaciones consisten en la combinación de, al menos, dos unidades léxicas cuyo vínculo está condicionado por ciertas restricciones combinatorias, que no siempre son compartidas entre distintas lenguas. De esta forma, un sustantivo -por ejemplo, gemitus 'gemido' - se asocia de forma regular con un determinado verbo (o un número reducido de ellos) -caso del verbo dare (gemitum dare 'dar un gemido, gemir'), aunque «desde el punto de vista sintáctico-semántico pudiera realizarse otra selección» (Írsula, 1994: 279).

Así las cosas, los ejercicios que se muestran a continuación buscan familiarizar al alumnado con la noción de restricción combinatoria. Para ello, nos serviremos de la información contenida en los diccionarios $(\$ 3.1 .1)$ y de los propios textos latinos junto con sus traducciones en español $(\$ 3.1 .2)$.

\subsubsection{Actividades basadas en el uso del diccionario}

Un recurso que nos puede ayudar a identificar combinaciones frecuentes y preferentes de palabras en latín es la información contenida en los diccionarios. 
De forma general, en la entrada de un determinado lema encontramos, además de sus distintas definiciones, las palabras que de manera recurrente aparecen en su contexto más cercano. Así, por ejemplo, en la entrada del sustantivo castra en el Vox, podemos ver la siguiente información combinatoria: castra ponere, acampar, sentar los reales; castra munire, communire, constituere, construir un campamento, fortificarlo; castra movere, levantar el campo, etc. (Vox, $21^{\text {a }}$ ed., s.v. 'castra').

Proponemos las siguientes actividades basadas en la información que recogen los diccionarios?:

a. Buscar en la entrada del verbo habeo todos los sustantivos en caso acusativo con los que se combina, hacer con ellos una lista y escribir el significado que adquieren juntos; por ejemplo, odium habere in aliquem 'tener odio a uno'.

b. Buscar en la entrada de los sustantivos metus y spes todos los verbos con los que se combinan, listarlos y escribir el significado que adquieren juntos; por ejemplo, metum capere 'tener miedo'.

c. Buscar en el diccionario las colocaciones que se ofrecen en la siguiente Tabla 1, teniendo en cuenta que estas pueden aparecer o bien en la entrada del sustantivo o bien en la del verbo. Indicar en la tabla dónde se documentan (nombre y/o verbo) y consignar su traducción.

\begin{tabular}{|l|c|c|c|}
\hline \multicolumn{1}{|c|}{ Colocaciones } & Nombre & Verbo & Traducción \\
\hline Verbum facere & $\mathrm{X}$ & & Hablar, arengar \\
\hline Saltum dare & & & \\
\hline Iter facere & & & \\
\hline Impetum facere & & & \\
\hline Virtutem habere & & & \\
\hline Suspicionem habere & & & \\
\hline Damnum dare & & & \\
\hline
\end{tabular}

Tabla 1. Modelo para la actividad basada en la información que aporta el diccionario.

\subsubsection{Actividades basadas en textos latinos y sus traducciones}

Para que los estudiantes entren en contacto con estas combinaciones restringidas de palabras, planteamos como actividad reconocer algunas de las colocaciones mencionadas en el ejercicio anterior en diferentes pasajes de autores clásicos. Ofrecemos los siguientes textos a manera de ejemplo:

${ }^{9}$ Para estudiantes de Bachillerato podría utilizarse el diccionario Vox, para niveles universitarios iniciales, el Diccionario latino-español de Blánquez, el Dictionnaire Latin Français de Gaffiot y el Oxford Latin Dictionary. 
(4) uulneribus didicit miles habere metum (Prop. 3.11.6)

(5) Ita dies circiter quindecim iter fecerunt, ut inter nouissimum hostium agmen et nostrum primum non amplius quinis aut senis milibus passuum interesset (Caes. Gall. 1.15.4-5)

(6) Quamquam istud mihi erit molestum triduom, et damnum dabis, faciam (Plaut. Cist. 106-107)

Como complemento a esta actividad, se puede solicitar a los alumnos que consulten al menos una traducción de estos textos y la comparen con sus respectivos originales. El objetivo aquí es que comprendan que no siempre hay una correspondencia exacta entre las colocaciones latinas y sus equivalentes en castellano, tal como se observa en las traducciones que presentamos a continuación y se especifica entre paréntesis:

(7) El soldado aprendió a causa de las heridas a tener miedo (habere metum - 'tener miedo' $)^{10}$.

(8) Así caminaron durante unos quince días, de manera que entre la retaguardia de los enemigos y nuestra vanguardia no hubiera más de cinco o seis mil pasos (iter facere - 'caminar').

(9) Aunque esos tres días me serán molestos y me causarás un daño, lo haré (damnum dare - 'causar un daño').

\subsection{ESTUDIO DE LAS COLOCACIONES DESDE UNA PERSPECTIVA SEMÁNTICA}

La segunda parte de nuestra propuesta se centra en el estudio de las colocaciones desde el punto de vista de su significado. Para ello, nos basamos en el trabajo que Gross $(1994,2008)$ ha desarrollado sobre combinaciones verbo-nominales en francés; según este autor, existe una distribución entre tipos de sustantivos que comparten ciertos rasgos de significado y la selección que hacen de un determinado verbo soporte ${ }^{11}$.

Por una parte, Gross propone que los nombres de Estado - del tipo peur 'miedo' o soif 'sed' - se combinan en francés con el verbo avoir 'tener' para formar colocaciones; del mismo modo, en latín, nombres que describen Estados como dolorem, timorem y memoriam ('dolor', 'temor' y, con ciertos matices, 'recuerdo') se combinan preferentemente con habere 'tener'.

Por otra, este autor relaciona los nombres de Acción con el verbo faire 'hacer' - faire un voyage 'hacer un viaje', faire l'ascension 'escalar'-; de nuevo, esta conclusión puede aplicarse al latín: nombres como irruptionem 'incursión', proelium 'combate' o rebellionem 'rebelión' suelen aparecer frecuentemente en colocaciones con facere 'hacer'.

${ }^{10}$ A partir de este punto, todas las traducciones que se ofrecen son propias.

${ }^{11}$ Esta distribución se encuentra motivada, en algunos casos, por cierta afinidad semántica entre nombres predicativos y verbos soporte; algunas reflexiones al respecto se encuentran en Penadés (2017). 
Además, de manera tangencial, Gross señala que existe un grupo específico de nombres de Acción que, en lugar de faire, eligen formar colocaciones con el verbo donner 'dar'; se trata de los sustantivos que hacen referencia a golpes, como gifle 'bofetada' en donner une gifle 'dar una bofetada', o coup 'golpe' en donner un coup 'dar un golpe' ${ }^{\prime 2}$. Esta distribución, una vez más, es extrapolable al latín: como algunos autores han señalado ( $c f$. Martín, 1996, 2018), también nombres como uerbera latigazos' o morsum 'mordisco' seleccionan generalmente el verbo dare 'dar'. Asimismo, podemos incluir en esta categoría algunos sustantivos que designan tipos de movimiento (saltum 'salto', staticulos 'pasos de danza', etc.) (Alonso, 2015; Pompei, 2018) y sonidos (sonitum 'sonido', gemitum 'gemido') (Koike, 2001; Martín, 1996, 2018).

La anterior distribución se resume para el latín en la Tabla 2:

\begin{tabular}{|c|c|c|}
\hline $\begin{array}{c}\text { Nombres de Estado } \\
+ \text { habere }\end{array}$ & $\begin{array}{c}\text { Nombres de Acción } \\
+ \text { facere }\end{array}$ & $\begin{array}{c}\text { Nombres de golpes, } \\
\text { tipos de movimiento } \\
\text { o sonidos + dare }\end{array}$ \\
\hline Dolorem & Irruptionem & Verbera \\
\hline Timorem & Proelium & Saltum \\
\hline Memoriam & Rebellionem & Sonitum \\
\hline
\end{tabular}

Tabla 2. Relación entre tipos de nombres y verbos soporte en latín.

A partir de esta explicación y con el objetivo de que los estudiantes reconozcan la relación existente entre tipos de nombres y los verbos soporte con los que se combinan, proponemos desarrollar en el aula tres actividades. La primera actividad consiste en i) identificar en una serie de pasajes los verbos facere, habere y dare y sus complementos en acusativo; ii) establecer si estos complementos pertenecen a uno de los tres tipos de nombres explicados (Estado, Acción y golpes/tipo de movimiento/sonidos); y iii) determinar si estas combinaciones de verbo y nombre son colocaciones. Presentamos la Tabla 3 como modelo:

\begin{tabular}{|c|c|c|c|c|}
\hline Pasaje & Identificación & Verbo & $\begin{array}{l}\text { Tipo de } \\
\text { nombre }\end{array}$ & Colocación \\
\hline $\begin{array}{l}\text { Reperiebat etiam in quaerendo Caesar, quod } \\
\text { proelium equestre aduersum paucis ante diebus } \\
\text { esset factum (...) (Caes. Gall. } 1.18 .9-10 \text { ) }\end{array}$ & proelium facere & Facere & $\begin{array}{l}\text { Nombre de } \\
\text { Acción }\end{array}$ & $\mathrm{X}$ \\
\hline $\begin{array}{l}\text { aptior illa quidem placido dare uerbera ponto } \\
\text { (Ou. epist. 18.23) }\end{array}$ & uerbera dare & Dare & $\begin{array}{l}\text { Nombre de } \\
\text { golpes }\end{array}$ & $\mathrm{X}$ \\
\hline confitemur cistellam habere (Plaut. Cist. 741) & cistellam habere & Habere & Otro & \\
\hline $\begin{array}{l}\text { et uitae necisque in suos habet potestatem } \\
\text { (Caes. Gall. 1.16.6) }\end{array}$ & $\begin{array}{l}\text { potestatem } \\
\text { habere }\end{array}$ & Habere & $\begin{array}{l}\text { Nombre de } \\
\text { Estado }\end{array}$ & $\mathrm{X}$ \\
\hline
\end{tabular}

Tabla 3. Modelo para la actividad de naturaleza semántica basada en la identificación de tipos de sustantivos.

${ }^{12}$ Encontramos esta misma correspondencia en español: sustantivos como patada, puñetazo, azote o golpe se combinan con el verbo dar (cf. Herrero, 2002; Koike, 2001). 
Por su parte, como segunda actividad presentamos un ejercicio de asociación de términos. En él, se pide a los estudiantes que relacionen los verbos facere, habere y dare, que aparecen en la primera columna de la Tabla 4, con los nombres de la segunda. Una vez terminado el ejercicio, sería conveniente que el docente incidiera en la relación semántica entre estos verbos y los tipos de sustantivos, y aportara una traducción para cada colocación.

\begin{tabular}{|c|c|}
\hline Verbo & Nombres \\
\hline \multirow{4}{*}{ Facere } & Murmur \\
\cline { 2 - 2 } & Saltum \\
\cline { 2 - 2 } & Laetitiam \\
\cline { 2 - 2 } & Conspirationem \\
\hline \multirow{4}{*}{ Habere } & Bellum \\
\cline { 2 - 2 } & Ludos \\
\cline { 2 - 2 } & Timorem \\
\hline \multirow{4}{*}{ Dare } & Deditionem \\
\cline { 2 - 2 } & Motus \\
\cline { 2 - 2 } & Curam \\
\cline { 2 - 3 } & Admirationem \\
\cline { 2 - 3 } & Aedificationem \\
\hline
\end{tabular}

Tabla 4. Modelo para la actividad de relación entre verbos soporte y distintos sustantivos.

El último ejercicio dentro de este bloque está constituido por una serie de pasajes de autores clásicos en los que aparecen nombres de Estado, de Acción y de golpes, tipos de movimiento o sonidos. Los estudiantes deberán completar el texto con el verbo soporte correspondiente (facere, habere o dare) en la forma que se especifica entre paréntesis:

(10) Sequentique die cum iter____ (infinitivo presente activo) Caesar cum copiis uellet (...) (Bell. Hisp. 28.1.1) (presente indicativo activo, $3^{a}$ persona singular) quoque iam saltus intra

(14) piloque posito stricto gladio in hostem impetum (presente indicativo activo, $3^{\mathrm{a}}$ persona caua texta carinae fluctus (Ou. met. 11.524-25) tum uero ingentem gemitum (presente indicativo activo, singular) pectore ab imo (Verg. Aen. 1.485) at uiri saepe excellentis ancipites uariique casus etitiam, molestiam, spem, $3^{\text {a }}$ persona plural) admirationem, exspectationem, laetitiam, molestiam, spem, timorem (Cic. fam. 5.12.5) activo, $3^{\text {a }}$ persona singular) (Liu. 7.16.5)

(15) ex quo perspicuum est, maiorem curam (presente indicativo marsuppii quam uitae nostrae (Varro Log. 2.1) (infinitivo presente activo) nos 


\subsection{LAS COLOCACIONES DESDE UN ENFOQUE SINTÁCTICO}

Antes hemos visto que el núcleo semántico de la colocación es el nombre, ahora explicaremos que también lo es a nivel sintáctico, siguiendo a Langer (2005):

«The fundamental idea of a support verb construction is the realisation of some or all arguments of the predicative noun in syntactic slots provided by the support verb. In prototypical support verb constructions, the verb does not semantically subcategorize any of its syntactic complements» (Langer, 2005: 184).

Desde esta perspectiva, analizaremos comparativamente los complementos de los verbos facere, habere y dare en combinaciones libres y en colocaciones. Veamos las oraciones (16) y (17) con facere:

(16) pontem fecit in Histro flumine, qua copias traduceret (Nep. Milt. 3.1)

"Construyó un puente en el río Histro por donde hacer pasar sus tropas»

(17) Sed Caesar (...) Silium legatum cum expedita manu irruptionem in Chattos facere iubet (Tac. ann. 2.7)

«Pero el César ordena al legado Silio hacer una incursión contra los Catos con una tropa ligera»

En una oración como la de (16), pontem 'puente' es un complemento de facere en su acepción o significado pleno 'construir'. En (17) la situación es un poco más compleja: en este caso facere no es sinónimo de 'construir' o 'crear', sino que se comporta como un verbo soporte del sustantivo predicativo irruptionem 'incursión'. Esto es evidente porque in Chattos 'contra los Catos' no puede asociarse fácilmente con facere, puesto que este verbo no suele presentar un sintagma preposicional -y menos con un valor espacial de dirección- como complemento. En cambio, irruptionem, como su verbo base irrumpo 'irrumpir', presenta típicamente un sintagma preposicional de dirección como argumento o complemento; así pues, in Chattos es seleccionado más por irruptionem que por facere. Parece claro, por tanto, que la estructura sintáctica de la colocación reposa en gran medida en la del nombre.

Ahora comparemos las oraciones (18) y (19) con el verbo habere:

(18) Ei ne noceret quam domi ante habui capram / neu discordarent, [ni]si ambae in uno essent loco (Plaut. Merc. 230-31)

«(...) para que no le hiciera daño la cabra que yo tenía desde antes en mi casa, ni se fueran a pelear si estuvieran las dos en el mismo lugar»

(19) (...) pro illo odio quod habuit in equestrem ordinem (Cic. Cluent. 151) «(...) por aquel odio que [Sila] tuvo hacia el orden ecuestre»

En (18), el complemento en acusativo capram 'cabra' resulta esperable con habere en su acepción como verbo de posesión: 'la cabra que yo tenía'. De nuevo, esto no es lo que sucede con el argumento in equestrem ordinem 'hacia el orden ecuestre' en la oración de (19). Una complementación como in + Acusativo no se relaciona con el verbo habere en su significado pleno; sin embargo, es bastante frecuente 
con el sustantivo odium 'odio' que es el referente de quod 'que' en la oración: 'el odio hacia alguien ${ }^{13}$.

Por último, analicemos las oraciones (20) y (21) con dare:

prandium uxor mihi perbonum dedit (Plaut. Most. 692)

«Mi esposa me ha dado un almuerzo excelente»

Dixit et e curru saltum dedit ocius aruis (Verg. Aen. 12.681)

«Dijo y rápidamente dio un salto desde el carro hacia la campiña»

En la oración (20) el verbo dare presenta su estructura de complementación prototípica: un Sujeto en nominativo (uxor 'esposa'), un Objeto en caso acusativo (prandium 'almuerzo') y un complemento Indirecto en dativo (mibi 'me, a mí'). Frente a esto, en (21) el complemento preposicional e curru 'desde el carro', que indica un punto de origen, se vincula fácilmente con el sustantivo de movimiento saltum 'salto'. Otra vez, los complementos de la oración parecen depender más del sustantivo predicativo que del verbo.

Para afianzar los conocimientos relativos a la naturaleza sintáctica de estas construcciones en latín, planteamos la actividad que se describe a continuación: i) identificar en un grupo de oraciones con los verbos habere, facere y dare si, con excepción de los complementos en acusativo, hay otros complementos; ii) en caso de que sea así, establecer si estos se corresponden con la estructura argumental prototípica del verbo o si, por el contrario, lo hacen con la estructura del nombre en acusativo; y iii) a partir de los criterios anteriores, deducir si se trata de una colocación. La Tabla 5 sirve como modelo para esta actividad:

\begin{tabular}{|l|c|c|c|c|}
\hline \multicolumn{1}{|c|}{ Pasaje } & $\begin{array}{c}\text { Complementación } \\
\text { diferente al } \\
\text { acusativo }\end{array}$ & $\begin{array}{c}\text { Asociada } \\
\text { al verbo }\end{array}$ & $\begin{array}{c}\text { Asociada } \\
\text { al nombre }\end{array}$ & Colocación \\
\hline $\begin{array}{l}\text { (...) si in nostros fines impetum faceret } \\
\text { (Caes. Gall. 1.44.8) }\end{array}$ & in nostros fines & $\begin{array}{c}\mathrm{x} \\
\text { (impetum) }\end{array}$ & $\mathrm{x}$ \\
\hline $\begin{array}{l}\text { Iugurtham magnificum ex Albini socordia } \\
\text { spem salutis in solitudine aut fuga coegis- } \\
\text { set habere (Sall. Ing. 55.1) }\end{array}$ & $\begin{array}{c}\text { in solitudine } \\
\text { aut fuga }\end{array}$ & $\begin{array}{c}\mathrm{x} \\
(\text { spem })\end{array}$ & $\mathrm{x}$ \\
\hline $\begin{array}{l}\text { Sic cursum in medios rapidus dedit (Verg. } \\
\text { Aen. 10.870) }\end{array}$ & in medios & $\begin{array}{c}\mathrm{x} \\
\text { (cursum) }\end{array}$ & $\mathrm{x}$ \\
\hline Dotes filiabus suis non dant (Cato orig. 94) & filiabus suis & $\begin{array}{c}\mathrm{x} \\
\text { (dant) }\end{array}$ & & \\
\hline
\end{tabular}

Tabla 5. Modelo para la actividad de naturaleza sintáctica basada en la estructura de complementación del sustantivo.

${ }^{13}$ De hecho, el Oxford Latin Dictionary recoge este tipo de complementación en la entrada del sustantivo odium. Asimismo, $c f$. Tur (2019). 
Por último, con el objetivo de que los estudiantes consoliden e integren las explicaciones teóricas vistas y llevadas a la práctica hasta este punto (respecto de la restricción combinatoria, la semántica y la sintaxis de las colocaciones latinas), ofrecemos el siguiente ejercicio. Este consiste en unir los verbos de la primera columna de la Tabla 6 con los sustantivos de la segunda para formar colocaciones y, a continuación, elegir un complemento de la tercera columna que se ajuste semántica y sintácticamente a ellas.

\begin{tabular}{|c|c|c|}
\hline Verbos soporte & Sustantivos & Complementos \\
\hline \multirow{2}{*}{ Habere } & Dolorem & Pectore \\
\cline { 2 - 3 } & Saltum & In Asiam \\
\hline \multirow{2}{*}{ Facere } & Gemitum & Ex curru \\
\cline { 2 - 3 } & Impetum & In uirtute ducis \\
\hline \multirow{2}{*}{ Dare } & Iter & In hostem \\
\cline { 2 - 3 } & Spem & Ab partu \\
\hline
\end{tabular}

Tabla 6. Modelo para la actividad de relación entre verbos soporte, sustantivos y complementos.

Es el momento ahora de recopilar los principales aportes de este artículo.

\section{CONCLUSIONES}

A lo largo de este artículo hemos desarrollado una propuesta didáctica para abordar las colocaciones verbo-nominales en latín -del tipo de saltum dare 'dar un salto'-. Consideramos este un tema especialmente relevante porque, tal como hemos puesto en evidencia en $\$ 1$, ni las gramáticas latinas ni los libros de texto analizados dan cuenta de ellas de forma específica, pese a que son construcciones relativamente frecuentes en esta lengua.

A partir de esta constatación, hemos comenzado por definir el fenómeno de las colocaciones poniendo el foco en las restricciones combinatorias y en el grado de fijación que presentan. A continuación, hemos descrito algunos rasgos de estas construcciones para, luego, ahondar en el que, a nuestro juicio, es más pertinente para explicar y reflexionar sobre ellas en el aula: la inversión de la tarea de predicar, es decir, la particularidad de que en este tipo de combinaciones es el sustantivo, y no el verbo, el núcleo semántico y sintáctico del conjunto $(\$ 2)$.

Nuestra propuesta didáctica propiamente dicha se inicia con una serie de ejercicios que trabajan sobre el concepto de restricción combinatoria $(\$ 3.1)$. El objetivo de esta primera fase es familiarizar a los estudiantes con este tipo de estructuras, utilizando para ello los diccionarios y los textos clásicos.

Tras este primer acercamiento, hemos desarrollado una propuesta de estudio de las colocaciones latinas basada en su significado. En este sentido, hemos retomado la idea de Gross $(1998,2004)$ de que cierto tipo de sustantivos relacionados entre sí semánticamente seleccionan un mismo verbo soporte: así, los nombres de Estado en latín (del tipo de timorem 'temor') tienden a combinarse con el verbo polisémico 
habere, mientras que los de Acción (irruptionem 'incursión') lo hacen con facere, y un último grupo caracterizado por referir golpes, tipos de movimiento o sonidos (como uerbera 'latigazos', saltum 'salto' o gemitum 'gemido'), con el verbo dare. Es precisamente esta explicación la que guía las tres actividades de esta sección (\$3.2).

En cuanto a la sintaxis, hemos partido de la idea sugerida por diversos estudiosos y en particular por Langer (2005) de que los complementos de las colocaciones se relacionan más con el sustantivo que con el verbo (por ejemplo, el complemento in Chattos 'contra los Catos' de la colocación irruptionem facere 'hacer una incursión' parece estar más vinculado con irruptionem que con facere). Este rasgo orienta las dos actividades propuestas en este bloque, aunque para el desarrollo de la última se hace preciso integrar todos los conocimientos adquiridos (\$3.3).

Para terminar, quisiéramos subrayar la idea de que todas las actividades presentadas son una muestra representativa de las que se podrían desarrollar en el aula.

RECIBIDO: marzo 2021; ACEPTADO: abril 2021.

\section{REFERENCIAS BIBLIOGRÁFICAS}

Alonso, Margarita (2004): Las construcciones con verbo de apoyo, Visor Libros, Madrid.

Alonso, Zoa (2015): «La expresión latina motus dare. ¿Una colocación con verbo soporte?», en M. T. Muñoz - L. Carrasco (eds.), Miscellanea latina, Sociedad de Estudios Latinos, Madrid, pp. 219-225.

BAN̄os, José Miguel (coord.) (2009): Sintaxis del latín clásico, Liceus, Madrid.

BAÑos, José Miguel (2012): «Verbos soporte e incorporación sintáctica en latín: el ejemplo de ludos facere», Revista de Estudios Latinos (RELat) 12: 37-57.

BAÑos, José Miguel (2016): «Las construcciones con verbo soporte en latín: sintaxis y semántica», en E. Borrell - P. Gómez (eds.), Omnia mutantur, Universitat de Barcelona, Barcelona, pp. 15-39.

BAÑos, José Miguel y JimÉnEZ, María Dolores (2017): «Arrepentirse’ en el Nuevo Testamento en griego y en latín: el empleo de las construcciones con verbo soporte en la Vulgata», Cuadernos de Filología Clásica. Estudios Latinos 37 (1): 11-32.

BASSOLS, Mariano ([1956] 1992): Sintaxis Latina, Consejo Superior de Investigaciones Científicas, Madrid.

BLÁNQUeZ, Agustín (1985): Diccionario Latín-Español, 3 vols., Sopena, Barcelona.

BODElot, Colette y SPEVAK, Olga (2018) (dirs.): Les constructions à verbe support en latin, Cahiers du Laboratoire de Recherche sur le Langage, 7, Presses Universitaires Blaise Pascal, ClermontFerrand.

De Miguel, Elena (2006): «Tensión y equilibrio semántico entre nombres y verbos: el reparto de la tarea de predicar», en M. VILLAYANDre (ed.), Actas del XXXV Simposio Internacional de la Sociedad Española de Lingüistica, Universidad de León, León, pp. 1289-1313.

EDEBÉ (2011): Latín I Bachillerato, Edebé, Barcelona.

GafFiot, Félix (1934): Dictionnaire Latin-Français, Hachette-Livre, Paris.

García Gual, Carlos; Andrés, Mariano y Monge, José Antonio (2010): Latín 1 Bachillerato, Santillana Educación, Madrid. 
GARZÓn, Eveling y JimÉnEZ, María Isabel (2018): «Quaestio: un análisis de la eventividad nominal a partir de sus colocaciones funcionales» en C. BODELOT - O. SPEVAK (dirs.), Les constructions à verbe support en latin, Cahiers du Laboratoire de Recherche sur le Langage, 7, Presses Universitaires Blaise Pascal, Clermont- Ferrand, pp. 53-75.

GLare, P.G.W. (1968): Oxford Latin Dictionary, Clarendon Press, Oxford.

Gross, Gaston (1994): «Classes d'objets et description des verbes», Langages 115: 15-30, doi: https://doi.org/ 10.3406/lgge.1994.1684.

Gross, Gaston (2008): «Les classes d'objets », Lalies 28: 111-165.

Gross, Maurice (1981): «Les bases empiriques de la notion de prédicat sémantique», Langages 63: 7-52, doi: https://doi.org/10.3406/lgge.1981.1875.

Herrero, José Luis (2002): «Los verbos soportes: el verbo dar en español», en M. GoNZÁLEZ, M. SouTo, A. VeIGA (coords.), Léxico y gramática, Tris Tram, Lugo, pp. 189-202.

Higueras, Marta (1996): «Aprender y enseñar léxico», en L. Miguel - N. SANS (coords.), Didáctica del español como lengua extranjera, 3, Colección Expolingua, Fundación Actilibre, Madrid, pp. 111-126.

Hofmann, Johann Baptist (1958): El latín familiar (traducido y anotado por J. Corominas), Instituto Antonio de Nebrija, Madrid.

ÍRSULA, Jesús (1994): «¿Entre el verbo y el sustantivo, quién rige a quién? El verbo en las colocaciones sustantivo-verbales» en A. Endruschat, M. Vilela, G. WotjaK (eds.), Verbo e estruturas frásicas / Colóquio Internacional de Linguística Hispânica, Faculdade de Letras do Porto, Porto, pp. 277-286.

JiMÉnEZ, María Isabel (2016): Colocaciones y verbos soporte: semántica y sintaxis del verbo pono [Tesis Doctoral], Universidad Complutense de Madrid, Madrid.

JiméneZ, María Isabel y Melis, Chantal (2018): «Evolución de las colocaciones causativas emocionales del latín al español», Anuario de Letras. Lingüística y Filología 6 (2): 75-109.

KOIKE, Kazumi (2001): Colocaciones léxicas en el español actual: estudio formal y léxico-semántico, Universidad de Alcalá de Henares, Madrid.

LANGER, Stefan (2005): «A formal specification of Support Verb Constructions», en S. LANGER D. SCHNORBuSCH, Semantik im Lexikon, Narr, Tübingen, pp. 179-202.

LEWIS, Michael (2000): Teaching Collocation: Further Development in the Lexical Approach, Language Teaching Publications, Hove.

LOPEZ, Iván (2019): «Las colocaciones verbo-nominales en griego y en latín: una comparación entre César y Jenofonte», Cuadernos de Filología Clásica. Estudios Latinos 39 (2): 209-225.

Martín, Antonio María (1996): «Dare, auxiliaire lexical en latin», en FruYT, M. - Moussy, C. (eds.), Structures lexicales du latin: actes de la table ronde du VII Colloque, Presses de l'Université de Paris-Sorbonne, Paris, pp. 149-168.

MarTín, Antonio María (2018): «Les emplois de dare comme verbe support: une réévaluation», en C. Bodelot - O. SPEVAK (dirs.), Les constructions à verbe support en latin, Cahiers du Laboratoire de Recherche sur le Langage, 7, Presses Universitaires Blaise Pascal, Clermont-Ferrand, pp. 149-167.

MeL'CuK, Igor (1995): «Phrasemes in Language and Phraseology in Linguistics», en M. EveraerT E. J. Van der Lienden (eds.), Idioms: Structural and Psychological Perspectives, Lawrence Erlbaum Associates, Hillsadle - Hove, pp. 167-232. 
MENDÓZAR, Juan (2015): «Causatividad y construcciones con verbo soporte en latín: el ejemplo de poena afficere», Cuadernos de Filología Clásica. Estudios Latinos 35 (1): 7-28.

MENDÓZAR, Juan (2020): «Las construcciones con verbo soporte en latín. Estado de la cuestión», Tempus 47: 7-48.

PENADÉs, Inmaculada (2017): «Arbitrariedad y motivación en las colocaciones», Revista de Lingüistica Teórica y Aplicada 55 (2): 121-142.

Pinkster, Harm (2015): The Oxford Latin Syntax. Volume 1: The Simple Clause, Oxford University Press, Oxford.

PompeI, Anna (2016): «Construction Grammar and Latin: the case of habeo», Pallas 102: 99-108, doi: https://doi.org/10.4000/pallas.3601.

POMPEI, Anna (2018): «Facere saltum ou dare saltum? Verbes supports et noms de mouvement», en C. BODELOT - O. SPEVAK (dirs.), Les constructions à verbe support en latin, Cahiers du Laboratoire de Recherche sur le Langage, 7, Presses Universitaires Blaise Pascal, Clermont-Ferrand, pp. 169-186.

RUBIO, Lisardo (1982): Introducción a la sintaxis estructural del latín, Ariel, Madrid-Caracas-México.

SANCHIS, Josefa (2012): Latín 1 Bachillerato, McGraw-Hill, Madrid.

TUR, Cristina (2019): Sintaxis y semántica de los nombres de sentimiento en latín: empleos adverbales $y$ colocaciones [Tesis Doctoral], Universidad Complutense de Madrid, Madrid.

TuR, Cristina (2020): «Una aproximación a la enseñanza de las colocaciones latinas a través de metáforas cognitivas», EClás. 158: 123-140.

VARRÓN (1990): De Lingua Latina. Introducción, traducción y notas de Manuel Antonio Marcos Casquero, Ministerio de Educación y Ciencia y Anthropos, Madrid-Barcelona.

VOX (2002): Diccionario Ilustrado Latín. Latino-Español, Español-Latino, 21 a ed., Spes Editorial, Barcelona. 
